\title{
MSFDR Antenna as a Logo for Smart Wearable Systems
}

\author{
Adithya Gaurav Singh ${ }^{\# 1}$, Ayush Gupta ${ }^{\# 2}$, A. K. Arya ${ }^{\# 3}$ \\ \#1-\#3 Department of Electronics \& Communication Engineering, \\ College of Technology, G.B.P.U.A.\&T., Pantnagar-263145, US Nagar, Uttarakhand, India \\ 1.agaurav51@gmail.com \\ 2 ayushguptaBT@gmail.com \\ 33arya@gbpuat.ac.in
}

\begin{abstract}
In this paper a Micro-Strip Fed Dielectric Resonator (MSFDR) Antenna is modified to be implemented in the form of a textile wearable logo for wireless communications in smart wearable systems. Over the years of extensive research DRAs have been found to be very effective in small size and wide bandwidth applications. Also in context to protruding nature of DRA it is an attractive choice for an application as a logo in clothing apparels. The proposed design study was carried out by using the Ansoft HFSS 13.0, a full wave EM software. The antenna operates on a wide frequency range from 5- 6.24 GHz. Analysis has been performed on four different textile substrates namely Wash Cotton, Jeans, Polycot and Polyester materials. Similar performance in terms of $\left|S_{11}\right| \mathrm{dB}$ and radiation patterns were observed with all four substrates and discussed in detail.
\end{abstract}

Keyword - Microstrip feeding, Dielectric Resonator Antenna, Wireless Communication, Smart Wearable Systems, Textile Substrates.

\section{INTRODUCTION}

Over the years profound research has been conducted to investigate the application of textile materials in fabrication of antennas which will prove to be crucial in manufacturing of reliable and unobtrusive smart wearable systems [1]-[6]. As observed in [7] these smart wearable systems will be instrumental in medical monitoring, tracking, navigation and military applications. Various modifications of microstrip antennas such as E-shaped patch antenna[8] and T-slotted patch antenna [9] have been utilized for wearable applications. Also compact accessorized wearable antennas integrated in button [10], jacket [11] and belt [12] have also been reported to have desirable performance characteristics. Another accessorized antenna in the form of logo has been investigated in [13] and is observed to have desirable performance characteristics but it is prone to distortions and bending due to human movements. This problem can be somewhat addressed by using a solid protruded Dielectric Resonator Antenna which is less vulnerable to bending and crumbling. Though DRAs have been used in wearable applications before [14] but not in a compact accessorized manner. Also the possibility of size reduction should also be investigated.

In this paper we propose a modified and reduced size DRA antenna design which can be easily integrated into regular clothing in the form of a logo or a button. The antenna uses a textile substrate. Simulation results in Ansoft HFSS 13.0 exhibit that antenna operates from $5.00 \mathrm{GHz}$ to $6.24 \mathrm{GHz}$ and is suitable for wide band applications. The antenna is studied for different textile substrates available in commercial market. Additionally, this paper contains 3D polar plot analysis for directivity and radiation pattern analysis for the gain. These results have been discussed in following text extensively. In section II, materials for fabrication and methods for obtaining results are illustrated. This section discusses brief characteristics of DRA to get a clearer understanding of its working and the proposed antenna dimensions and the geometry. In section III, simulation results for the specified geometry are presented and also variations in antenna performance due to changes in various parameters have been observed. Section IV concludes the research work done in this paper along with future research work areas.

\section{MATERIALS AND METHODS}

Following text elaborates the technology and all the materials which would be necessary to fabricate the proposed antenna.

\section{A. Dielectric Resonator Antenna}

A Dielectric Resonator Antenna (DRA) is a low profile radio antenna mostly used for high frequency applications. It is very similar to a microstrip patch antenna in terms of radiation patterns and feeding mechanisms. Physically, DRA consists of a block of ceramic material of various shapes known as the dielectric resonator, of a high dielectric constant mounted on a substrate and a ground plane. The dimension of the DRA is proportional to $\lambda_{0} / \sqrt{ } \varepsilon_{\mathrm{r}}$ where $\lambda_{0}$ is the free-space wavelength and $\varepsilon_{\mathrm{r}}$ is the dielectric constant. Thus, by choosing a high value of $\varepsilon_{\mathrm{r}}(\approx 10)$, the size of the DRA can be significantly reduced. There is no inherent conductor loss in dielectric resonators. This leads to high radiation efficiency of the antenna. The operating 
bandwidth of a DRA can be varied over a wide range by suitably choosing resonator parameters. A simple Dielectric Resonator antenna is illustrated in Fig. 1. Different types of feeding mechanisms such as probe feed, microstrip line, aperture coupled feed can be used in a dielectric resonator antenna. Microstrip feed line should be used for better integration of the antenna in the clothing apparel.

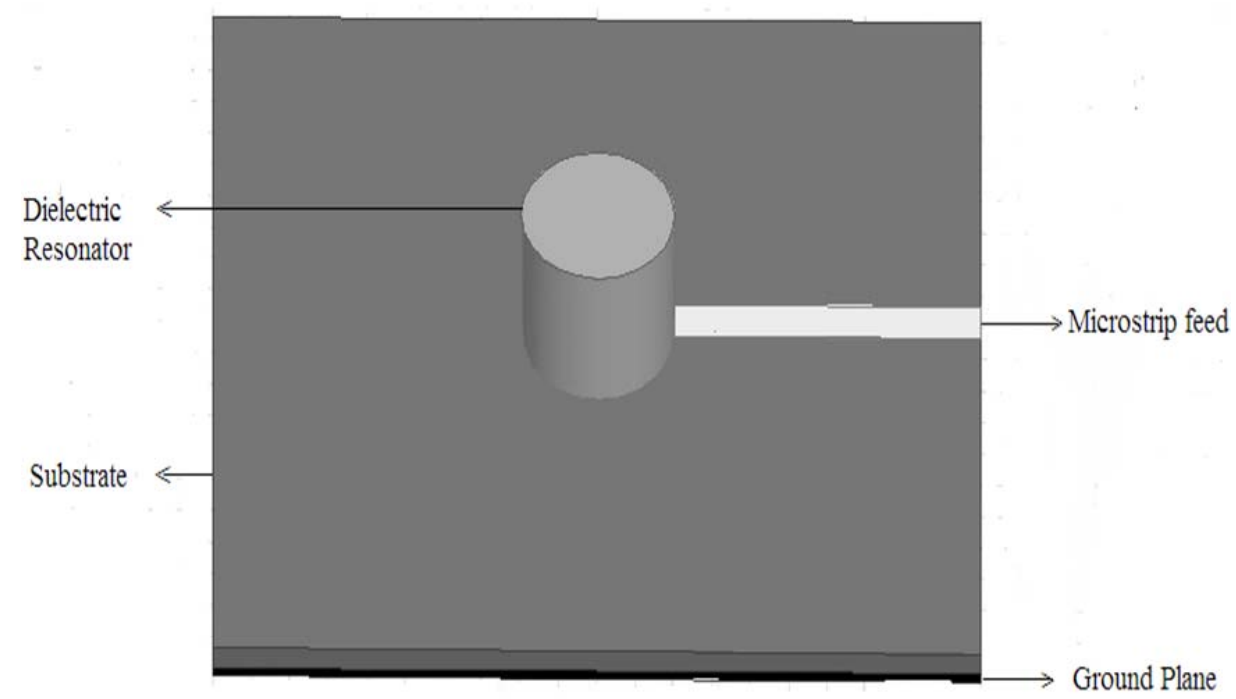

Fig. 1: A typical Dielectric Resonator Antenna b

DRA has a protruded type appearance which opens up the possibility for its utilization in fabricating designer type customizable antennas. This paper proposes a wearable logo antenna to exploit the above discussed property.

For the simulation of antenna, a full wave EM simulation software Ansoft HFSS 13.0 has been incorporated. Proper Boundary conditions have been taken care of and driven terminal solver has been employed to simulate the antenna. Microstrip feed line mechanism has been utilized. Substrate chosen for simulation is wash cotton which is easily available commercially and is also popular in textile industry. Dimensions of the substrate have been chosen such that they exceed the dimensions of ground plane as is evident in Table I. This was done to simulate the real world conditions, where textile material will be much larger in size than the ground plane, in a more precise manner. Following section elaborate the dimensions and material used for the fabrication of the antenna.

\section{B. Proposed Antenna Dimensions}

The dimensions for Antenna depicted in Fig. 2 are given in Table I. These dimensions were found to impart optimum performance characteristics to the antenna. The Dielectric Resonator is in the form of a notched cylinder which provides aesthetic value as well as is crucial for desirable performance characteristics. The diamond shaped notch is proposed to be made by slicing a corner of dimensions as stated above from a cube of the same material as mirctostrip feed line. This notch can then be attached to the dielectric resonator and the feed by an adhesive. As discussed in [14], this type notch is necessary for a better coupling between the feed and the dielectric resonator.

TABLE I. Different Antenna components and their dimensions

\begin{tabular}{|l|l|l|}
\hline S. No. & Antenna Component & Dimension \\
\hline 1. & $\begin{array}{l}\text { Notched up cylindrical dielectric } \\
\text { resonator }\end{array}$ & Height $=11 \mathrm{~mm} \mathrm{Width}=9 \mathrm{~mm}$ \\
\hline 2. & Microstrip Feed Line & Length $=10.75 \mathrm{~mm}$ Width $=6.8 \mathrm{~mm}$ \\
\hline 3. & $\begin{array}{l}\text { Diamond shape notch (Sliced } \\
\text { from a cube) }\end{array}$ & $\begin{array}{l}\text { Length }=6.56 \mathrm{~mm} \text { Width }=6.56 \mathrm{~mm} \\
\text { Height }=4.9 \mathrm{~mm}\end{array}$ \\
\hline 4. & Substrate & $\begin{array}{l}\text { Length }=44 \mathrm{~mm} \mathrm{Width}=43.7 \mathrm{~mm} \\
\text { Height }=1.6 \mathrm{~mm}\end{array}$ \\
\hline 5. & Ground plane & Length $=40 \mathrm{~mm} \mathrm{Width}=39.7 \mathrm{~mm}$ \\
\hline
\end{tabular}

Fig. 2 illustrates the expected design of the antenna which will be practically and commercially available. This antenna will be mounted on upper chest which is the popular site of logo in most of the clothing apparels. The notch in [14] has been used in the form of a rectangular strip. This paper proposes to use this notch in the shape of diamond. This modification will reduce the antenna dimensions thus making it more suitable for 
application as a textile antenna. Following figure elaborates the antenna geometry in a more theoretical way for better analysis.

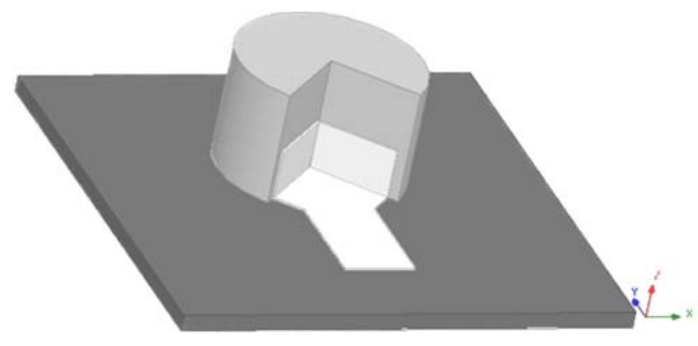

Fig. 2 : Schematic Antenna Design (perspective view)

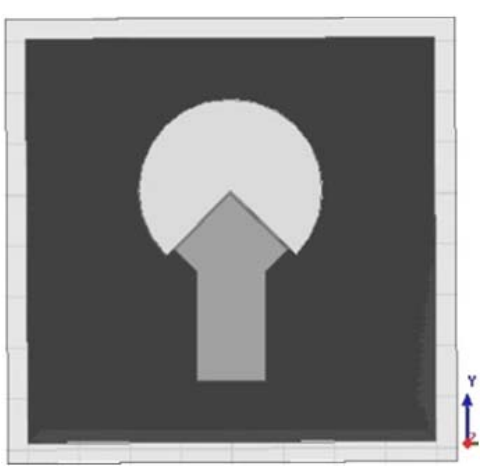

(a)

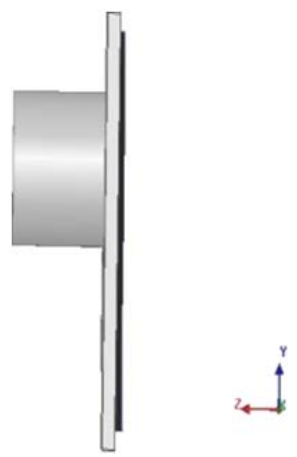

(b)

It is clear from Fig. 3, that the dimensions of substrate have been chosen such that they exceed the dimensions of the ground plane to evaluate the real world conditions in a better way. Table II defines all the materials proposed to be used in the different components of the antenna. Next section elaborates the results obtained after simulation of the antenna.

TABLE III. Different materials used for different components of the antenna

\begin{tabular}{|l|l|l|}
\hline S. No. & Antenna Component & Material Used \\
\hline 1. & $\begin{array}{l}\text { Notched up cylindrical dielectric } \\
\text { resonator }\end{array}$ & $\begin{array}{l}\text { Rogers RT/duroid 6010/6010LM }(\mathrm{tm}) \\
\left(\varepsilon_{\mathrm{r}}=10.2\right)\end{array}$ \\
\hline 2. & Microstrip feed line & Aluminium \\
\hline 3. & Diamond shape notch & Aluminium \\
\hline 4. & Substrate & Wash Cotton $\left(\varepsilon_{\mathrm{r}}=1.51\right)$ \\
\hline 5. & Ground plane & Copper \\
\hline
\end{tabular}

\section{III.RESULTS AND DISCUSSION}

Fig. 4 depicts the reflection coefficient or $\left|\mathrm{S}_{11}\right| \mathrm{dB}$ against frequency. It is the measure of how much power is reflected back at antenna port due to mismatch from the transmission line. Ideally, it should be as low as possible. Antenna is said to be operating in the range of frequency for which $\left|S_{11}\right|$ is less than $-10 \mathrm{~dB}$. It is evident from Fig. 4, that $-10 \mathrm{~dB}$ bandwidth ranges from $5.0 \mathrm{GHz}$ to $6.24 \mathrm{GHz}$ which is suitable for wide band communication.

\section{A. Optimized Results}

Fig. 5 illustrates the radiation patterns for frequencies $5.495 \mathrm{GHz}$ and $6.1 \mathrm{GHz}$, that have been observed in the Theta - plane for both $\mathrm{Phi}=0 \mathrm{deg}$ and $\mathrm{Phi}=90$ deg values. Desirable directional characteristics were observed. Another aspect of the antenna performance is its directivity. Directivity is the measure of the power density radiated in the direction of its strongest emission by the antenna, versus the power density radiated by a simple isotropic antenna radiating the same total power. Directivity of the antenna can analysed by studying the 3D polar plot. Fig. 6 depicts the 3D polar plot at $5.490 \mathrm{GHz}$.

Polar plot of the proposed antenna shows that the maximum emission is in the $+z$-direction and is minimal in -z-direction, hence the antenna, when used as a logo, will direct maximum amount of the radiation away from the body of user. This is a desirable characteristic as it will greatly reduce the radiation absorbed by the tissues of body of the user. 


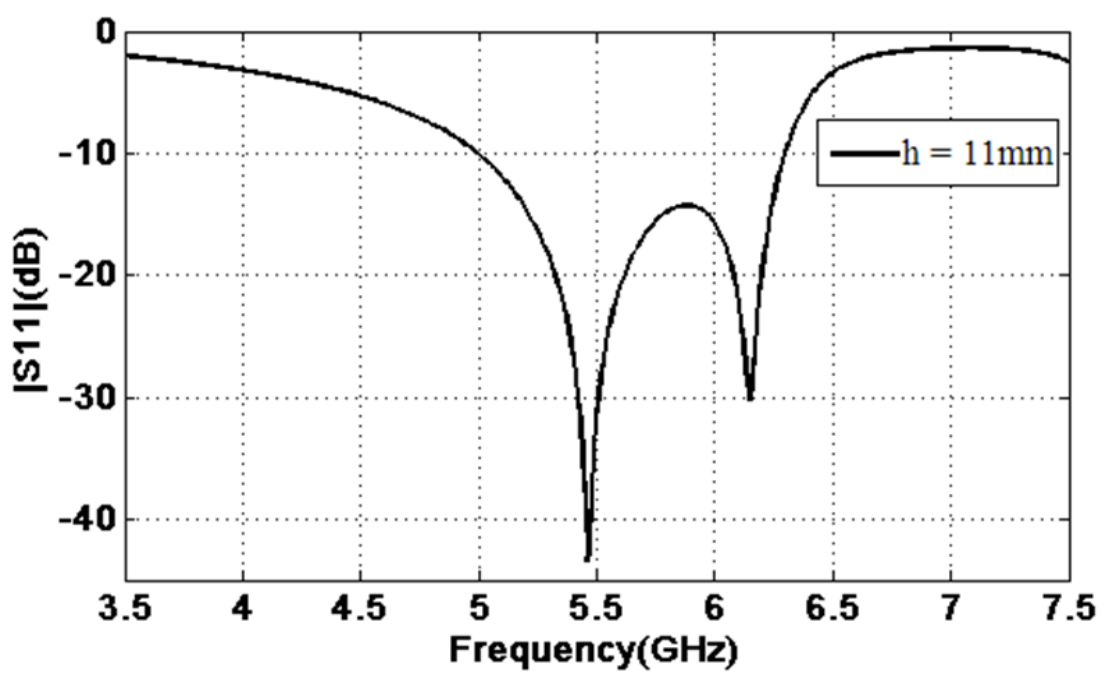

Fig. 4 : Proposed antenna $|\mathrm{S} 11| \mathrm{dB}$ parameter

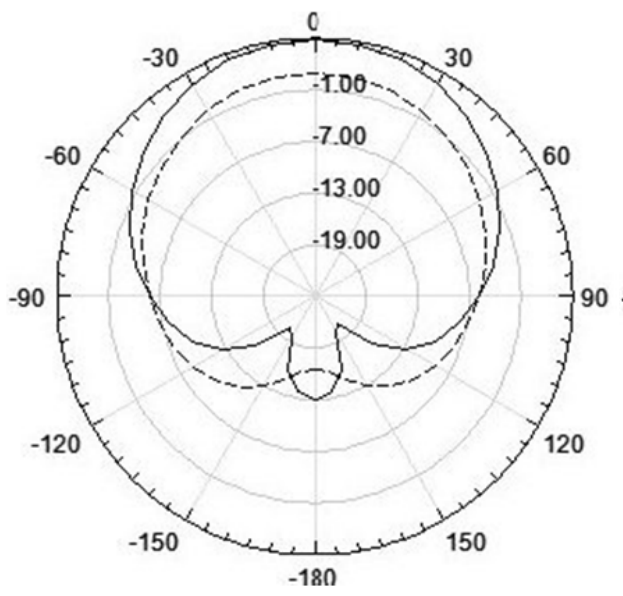

(a)

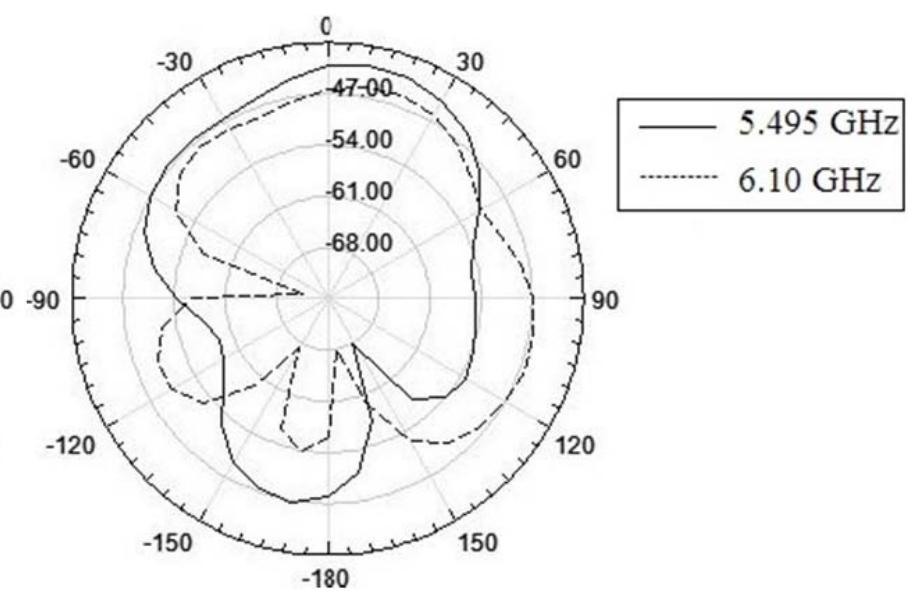

(b)

Fig. 5 Radiation Patterns at (a) $\mathrm{Phi}=0^{\circ}$ (b) $\mathrm{Phi}=90^{\circ}$
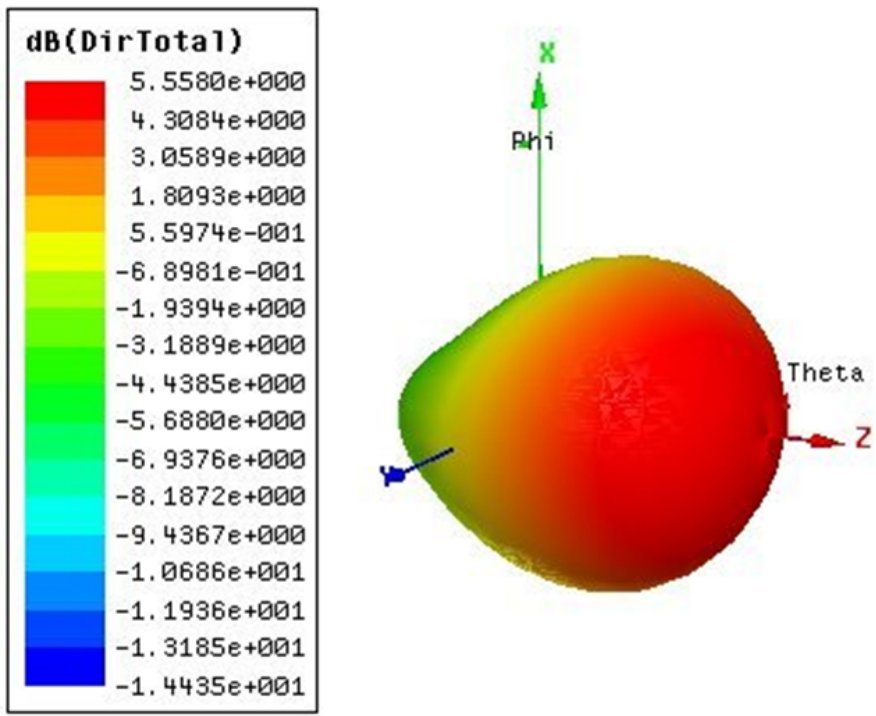

Fig. $6: 3 \mathrm{D}$ radiation pattern at $5.490 \mathrm{GHz}$ 


\section{B. Parameter Variation}

Further parametric analysis has been performed on the proposed antenna in this section. Different parameters such as height of the dielectric resonator, height of the diamond notch and substrate material were varied to observe the change in the antenna results. Fig. 7 shows how the antenna performance is expected to vary as the height of the dielectric resonator is varied. Simulations were performed for dielectric resonator height of $10 \mathrm{~mm}$, $11 \mathrm{~mm}$ and $12 \mathrm{~mm}$. All the other parameters such as notch height and substrate material were kept constant. Similarly, in Fig. 8 the simulations were performed for notch height of $4.8 \mathrm{~mm}, 4.9 \mathrm{~mm}$ and $5.0 \mathrm{~mm}$, keeping all other antenna parameters as constant. Parametric study is important in order to verify that the dimensions chosen earlier and results observed are in the most optimized form.

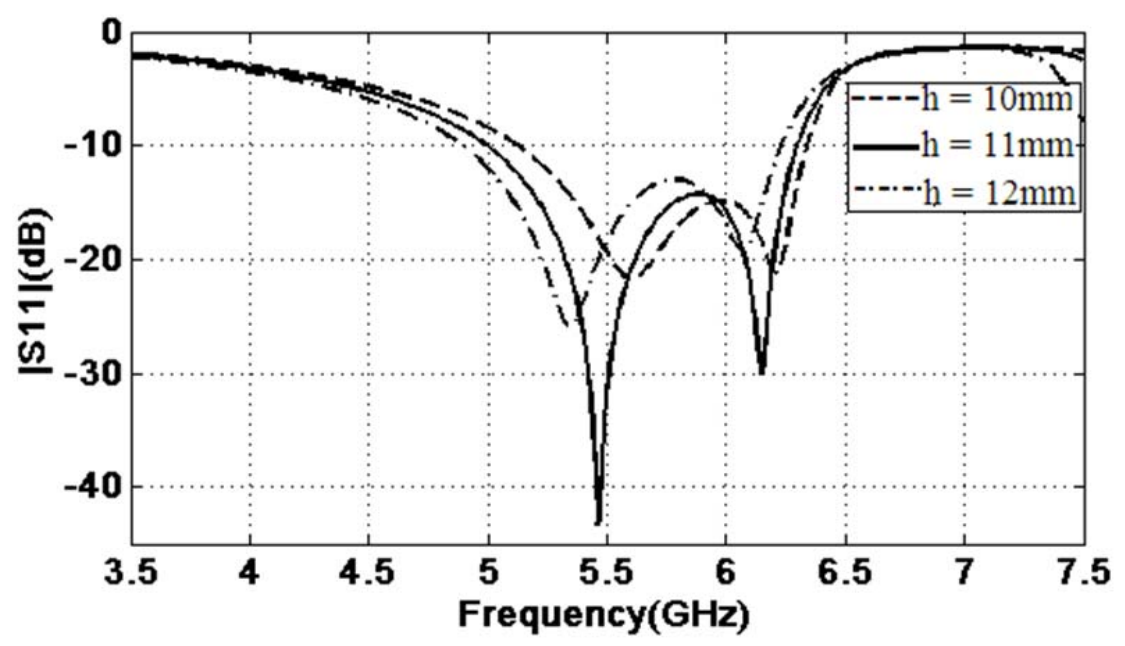

Fig. 7: Reflection coefficient for varying dielectric resonator height and all other parameters held constant

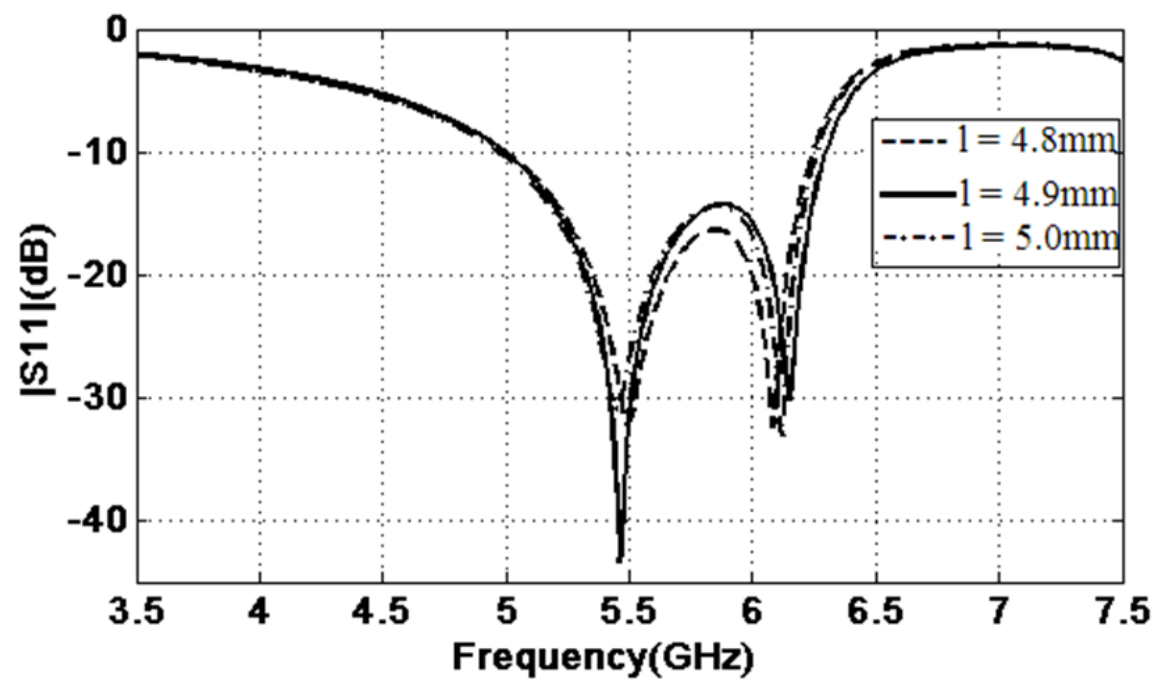

Figure 8 : Reflection coefficient for varying diamond notch height and all other parameters held constant

Both Fig. 7 and Fig. 8 make it exceedingly clear that antenna exhibits the best performance when height of the dielectric resonator and height of the diamond notch are $11 \mathrm{~mm}$ and $4.9 \mathrm{~mm}$ respectively. This proves that the dimensions prescribed in Table I are the optimized dimensions and the given antenna performs best for these specifications.

TABLE III. Dielectric Constants of the different textile materials analysed for the proposed antenna

\begin{tabular}{|l|l|l|}
\hline S. No. & Textile Material & Dielectric Constant \\
\hline 1. & Wash cotton & 1.51 \\
\hline 2. & Jeans & 1.67 \\
\hline 3. & Polycot & 1.56 \\
\hline 4. & Polyester & 1.44 \\
\hline
\end{tabular}

Antenna characteristics and their variations for substrate of different textile materials were also studied. Four of the most commercially available textile materials namely Wash Cotton, Jeans, Polycot, Polyester have been 
considered. Dielectric constants investigated for these materials in [15] have been used. The data in Table III has been illustrated in [15]. Simulation result for all these textile materials using the values of dielectric constant $\left(\varepsilon_{\mathrm{r}}\right)$ as specified in Table III is given in Fig. 9.

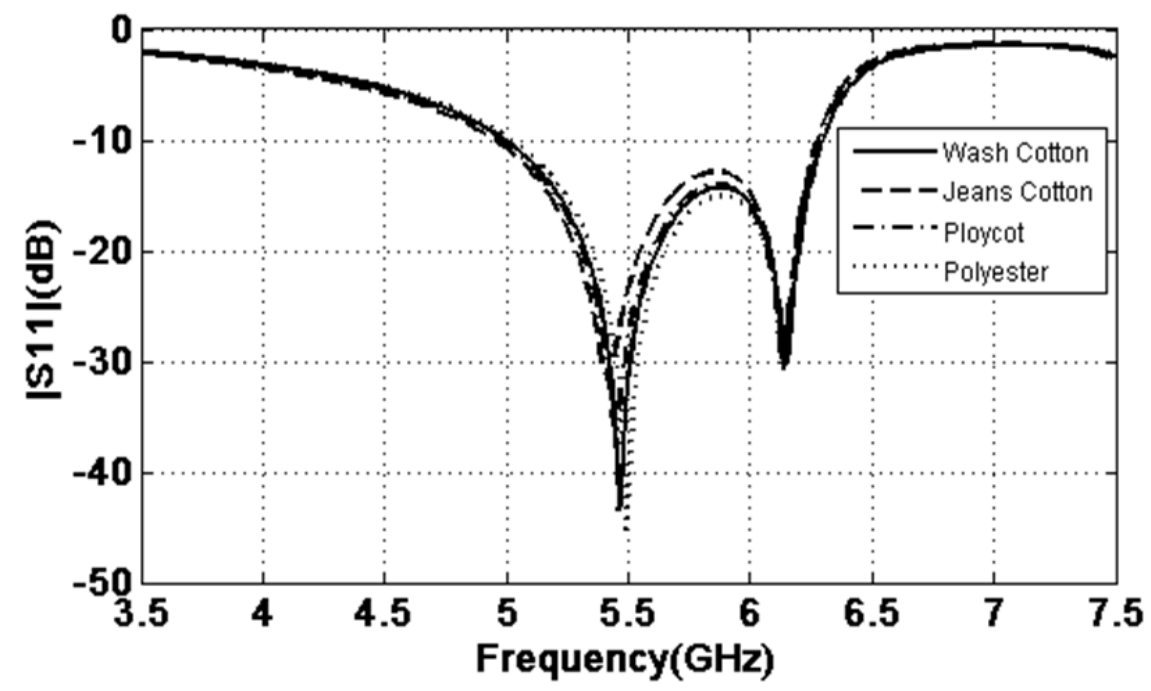

Fig. 9 : Reflection coefficient for Dielectric Resonator on different textile materials

The above stated results illustrate that the results for all considered textile materials are similar and resonant at approximately same frequencies. The best return loss of the four is observed for the substrate material described in Table II. i.e., wash cotton. A Return loss of $-48.3 \mathrm{~dB}$ was recorded. Even the worst case return loss recorded for Jeans substrate is $-31.5 \mathrm{~dB}$ which is still very desirable for application as a practical antenna. Another crucial observation from the above result is that the bandwidth of antenna in all four cases is approximately the same. This clearly indicates that the proposed antenna is widely applicable for different textile substrates and will prove to be an attractive alternative from a commercial point of view.

\section{IV.CONCLUSION}

After an extensive analysis of the simulation results, it can be concluded that DRA is a suitable alternative for wide band antenna applications in the form of a compact wearable logo in regular clothing apparels. Antenna performance for optimized geometry and materials show that antenna works efficiently for a range of frequencies from $5.00 \mathrm{GHz}$ to $6.24 \mathrm{GHz}$. This range of frequency is a part of C band and will cover WLAN band. It was also observed that antenna provides a bandwidth of approximately $1.24 \mathrm{GHz}$ which is highly desirable for wide band communication applications. A diamond notch type coupling mechanism was used for feeding which resulted in reduction of the antenna size making it more suitable for its integration as a logo. Simulations for four different substrate materials were performed, good results and a high degree of similarity was observed in all cases indicating that the proposed antenna will give a satisfactory performance for most of the textile substrates commercially available. Future research avenues for the above antenna will be the following:

- Different possible modifications in the antenna's geometry such as shape, material and cut out shape for the coupling notch.

- Different feeding techniques.

- Different notch shapes such as square, circular for coupling with microstrip feed line and the corresponding antenna behaviour.

- Further reduction of size and increase in bandwidth.

\section{REFERENCES}

[1] P. Salonen, F. Yang, Y. Rahmat-Samii, M. Kivikoski, WEBGA-Wearable Electromagnetic Band-Gap Antenna, 2004 IEEE Antennas and Propagation Society International Symposium, Vol. 1, pp. 451 - 454, Monterey, CA, USA, 2004.

[2] Salonen, P., Rahmat-Samii, Y., Hurne, H., and Kivikoski, M.: Dual-band wearable textile antenna'. Proc. of IEEE Antennas and Propagation Society Int. Symp., Monterey, CA, USA, June 2004, Vol. 1, pp. 463-466.

[3] C. Hertleer, H. Rogier and L. Van Langenhove, "A Textile antenna for Protective Clothing," IET Seminar Digest 2007 (11803), pp. 44-46, 2007.

[4] A. Tronquo, H. Rogier, C. Hertleer, and L. Van Langenhove. "A Robust Planar Textile Antenna for Wireless Body LANs Operating in the $2.45 \mathrm{GHz}$ ISM band", Electronics Letters, 42, pp. 142-143, (2006).

[5] Klemm, M., and G. Troester, "Textile UWB Antennas for Wireless Body Area Networks," IEEE Transactions on Antennas and Propagation, September 2005.

[6] R. Langley and Shaozhen Zhu, "Dual band wearable antenna," 2008 Loughborough Antennas and Propagation Conference, Loughborough, 2008, pp. 14-17. 
[7] B. Gupta, S. Sankaralingam and S. Dhar, "Development of wearable and implantable antennas in the last decade: A review," $201010^{\text {th }}$ Mediterranean Microwave Symposium, Guzelyurt, 2010, pp. 251-267.

[8] D. L. Paul, M. Klemm, C. J. Railton and J. P. McGeehan, "TEXTILE BROADBAND E-PATCH ANTENNA AT ISM BAND," 2007 IET Seminar on Antennas and Propagation for Body-Centric Wireless Communications, London, 2007, pp. 38-43.

[9] M. Abdullah and A. Khan, "Multiband wearable textile antenna for I.S.M body center communication systems," 2015 XXth IEEE International Seminar/Workshop on Direct and Inverse Problems of Electromagnetic and Acoustic Wave Theory (DIPED), Lviv, 2015, pp. 90-96.

[10] B. Sanz-Izquierdo, F. Huang and J. C. Batchelor, "Covert dual- band wearable button antenna," in Electronics Letters, vol. 42, no. 12 , pp. 668-670, 8 June 2006.

[11] B. Sanz-Izquierdo and J. C. Batchelor, "Wlan Jacket Mounted Antenna," 2007 International workshop on Antenna Technology: Small and Smart Antennas Metamaterials and Applications, Cambridge, 2007, pp. 57-60.

[12] B. Sanz-Izquierdo and J. C. Batchelor, "A Dual Band Belt Antenna," 2008 International Workshop on Antenna Technology: Small Antennas and Novel Metamaterials, Chiba, 2008, pp. 374-377.

[13] M. S. Mahmud and S. Dey, "Design, performance and implementation of UWB wearable logo textile antenna," 201215 International Symposium on Antenna Technology and Applied Electromagnetics, Toulouse, 2012, pp. 1-4.

[14] S. H. H. Mashhadi, Z. Wu and L. Z. Thamae, "Investigation of a wearable broadband textile Dielectric Resonator Antenna," 2010 Loughborough Antennas \& Propagation Conference, Loughborough, 2010, pp. 349-352

[15] S. Sankaralingam and B. Gupta, "Determination of Dielectric Constant of Fabric Materials and Their Use as Substrates for Design and Development of Antennas for Wearable Applications," in IEEE Transactions on Instrumentation and Measurement, vol. 59, no. 12, pp. 3122-3130, Dec. 2010. 